\title{
Comparison between the Conformations of Short Broken Chains and Stiff Chains
}

\author{
Hideatsu MAeda and Nobuhiko SAItô \\ Department of Applied Physics, Waseda University, Tokyo, 160, Japan. \\ Walter H. STOCKMAYER \\ Department of Chemistry, Dartmouth College, Hanover, N.H. 03755, U.S.A.
}

(Received October 13, 1970)

\begin{abstract}
A comparison is made of the second and the fourth moments of the end-to-end distances of several broken chain models with those of continous stiff chains. A shift factor $f$ is introduced in such a way that $n=f L D$, where $n$ is the degree of polymerization in the broken chain, $L$ is the contour length and $L / D$ is the mean square end-to-end distance in the limit of the long stiff chain. A single $f$-value is found to yield a good coincidence of both the second and the fourth moments of the end-to-end distance of the broken chain with those of the stiff chain. Values of the shift factor $f$ which make the best coincidence of the conformations of the broken chains and the stiff chain are 1.66 for the freely rotating chain, 10.49 for the polymethylene chain and 9.18 for the chain with independent hindrance-potentials of the polymethylene type.

KEY WORDS Stiff Chain / Wormlike Chain / Broken Chain / Conformation/Characteristic Ratio/Second and Fourth Moments of End-to-End Distance / Shift Factor /
\end{abstract}

The conformation of long flexible polymers is adequately represented by a Gaussian distribution in the absence of the excluded volume of the chain elements. This Gaussian conformation is the asymptotic one in the limit of a long Markovian chain; ${ }^{1,2}$ the mean square end-to-end distance $\left\langle\boldsymbol{R}^{2}\right\rangle$ is proportional to the degree of polymerization $n$, and the proportionality constant is characteristic of the chain under consideration. $^{3-5}$ In short chains or oligomers, however, the Gaussian approximation does not hold, and as $n$ increases the characteristic ratio $\left\langle\boldsymbol{R}^{2}\right\rangle / n b^{2}$ increases to reach finally a constant limiting value $C_{\infty}$.

The model conventionally adopted for representing flexible polymers is a broken chain model, in which free or restricted rotations about every bond are assumed by keeping the bond length $b$ and bond angle $\theta$ constant. On the other hand a differentiable space-curve is often convenient for describing semiflexible polymers and is sometimes called a stiff chain or wormlike chain. ${ }^{6,10}$ In this model the ratio of $\left\langle\boldsymbol{R}^{2}\right\rangle$ to the contour length $L$ behaves similarly to the ratio $\left\langle\boldsymbol{R}^{2}\right\rangle / \boldsymbol{n}$ in short broken chains.
In this paper comparisons between the conformations of short broken chains and stiff chains are given. Stiff chains are found to be a simpler model useful for describing the behavior of short chains in solution.

\section{SECOND AND FOURTH MOMENTS OF THE END-TO-END DISTANCE}

Recently great progress has been achieved in the statistical study of the conformations of various polymeric chains. For example, the conformation of the polymethylene chain was calculated by Nagai, ${ }^{14}$ Lifson, ${ }^{15}$ and Jernigan and Flory, ${ }^{4,11}$ on the assumption of interdependent internal rotations. The last-named authors compared their results for short polymethylene: chains with those of various models such as the freely jointed chain, freely rotating chain, chain. of independent rotations, and stiff chain. They concluded that none of these models adequately represents the conformational behavior of a realistic polymethylene chain. Our approach is somewhat different from that of Jernigan and Flory, and leads to a somewhat more favorable: 
conclusion regarding the utility of the stiff chain model.

One of our objectives is to establish the best method for relating the parameters of brokenchain models to those of the stiff chain. To do this, we first give here the expressions for the second and fourth moments of the end-toend distance $R$ for several chain models, all in the absence of excluded-volume effects.

(a) Stiff Chains

$$
\left\langle\boldsymbol{R}^{2}\right\rangle=\frac{L^{2}}{2(D L)^{2}}\left\{e^{-2 D L}-1+2 D L\right\} \quad \text { (1) } \quad\left\langle\boldsymbol{R}^{2}\right\rangle=b^{2}\left\{n \frac{1-\cos \theta}{1+\cos \theta}+2 \cos \theta \frac{1-(-\cos \theta)^{n}}{1+\cos \theta}\right\}
$$
persistence length.

$$
\begin{aligned}
\left\langle\boldsymbol{R}^{4}\right\rangle= & \frac{L^{4}}{2(D L)^{4}}\left\{\frac{10}{3} D^{2} L^{2}-\frac{52}{9} D L+\frac{107}{27}-4 e^{-2 D L}\right. \\
& \left.-2 D L e^{-2 D L}+\frac{1}{27} e^{-6 D L}\right\}
\end{aligned}
$$

where $L$ is the contour length and $1 / 2 D$ is the

(b) Freely Rotating Chain of Bond Length $b$

$$
\begin{aligned}
\left\langle\boldsymbol{R}^{4}\right\rangle= & b^{4}\left\{\frac{5}{3} n^{2}\left(\frac{1-\cos \theta}{1+\cos \theta}\right)^{2}-\frac{2 n(1-\cos \theta)\left(7-42 \cos \theta+3 \cos ^{2} \theta\right)}{9(1+\cos \theta)^{3}}\right. \\
& +\frac{8\left(2-40 \cos \theta+221 \cos ^{2} \theta-519 \cos ^{3} \theta+579 \cos ^{4} \theta-297 \cos ^{5} \theta+54 \cos ^{6} \theta\right)}{27(1+\cos \theta)^{4}(1-\cos \theta)^{2}(1-3 \cos \theta)} \\
& -\frac{8 \cos \theta\left(21 \cos ^{4} \theta+34 \cos ^{3} \theta-37 \cos ^{2} \theta+12 \cos \theta-2\right)}{(1+\cos \theta)^{4}(1-3 \cos \theta)^{2}}(-\cos \theta)^{n} \\
& -\frac{4 n\left(27 \cos ^{2} \theta+24 \cos ^{3} \theta-20 \cos \theta+1\right)(-\cos \theta)^{n}}{3(1+\cos \theta)^{3}(1-3 \cos \theta)} \\
& \left.+\frac{16\left(3 \cos ^{2} \theta-1\right)(1+3 \cos \theta)^{2}}{27(1+\cos \theta)^{4}(1-3 \cos \theta)^{2}}\left(\frac{2}{3 \cos ^{2} \theta-1}\right)^{-n}\right\}
\end{aligned}
$$

where $\theta$ is the angle between successive bonds, and in the numerical calculation it is put equal to the tetrahedral angle $\theta=109^{\circ}$, or $\theta=112^{\circ}$ from crystallographic data.

(c) Chain with Independent Hindrance-Potentials

In this model the internal rotations about each bond are assumed independent but are hindered by a symmetric potential. The limit for large $n$ of the characteristic ratio for this model was first calculated by Oka, ${ }^{12}$ and detailed studies on the second and the fourth moments of the end-to-end distance have been done by Volkenstein, ${ }^{3}$ Nagai, ${ }^{13}$ Jernigan, ${ }^{11}$ and Flory. ${ }^{11}$ In this paper we adopt Jernigan and Flory's work by assigning the valence angle $\theta=112^{\circ}$ instead of tetrahedral angle $109^{\circ}$, and the average rotating angle $\langle\cos \varphi\rangle=0.514$ to make the mean square end-to-end distance equal to 6.87 , the value consistent with both the experimental value and the calculated one by the model (d). In Figure 2 are shown the second and fourth moments of the end-to-end distance calculated by them.

\section{(d) Chains with Interdependent Rotations of Poly- methylene Type}

In this chain model, the rotations about each bond are not independent. Treatments for this model are discussed by many authors. ${ }^{3-5}$ We adopt the calculations of $\left\langle\boldsymbol{R}^{2}\right\rangle$ and $\left\langle\boldsymbol{R}^{4}\right\rangle$ given by Flory and Jernigan, ${ }^{11}$ but the results are not reproduced here. (See Figure 3)

\section{COMPARISON BETWEEN SHORT BROKEN CHAINS AND STIFF CHAINS}

The most important quantity associated with the conformation and characteristic of the molecular structure of a polymer chain is the limit of the characteristic ratio $\left\langle\boldsymbol{R}^{2}\right\rangle / n b^{2}$ for large $n$. We put for a broken chain

$$
\lim _{n \rightarrow \infty} \frac{\left\langle\boldsymbol{R}^{2}\right\rangle}{n b^{2}}=C_{\infty}
$$

and the corresponding quantity for a stiff chain is

$$
\lim _{L \rightarrow \infty} \frac{\left\langle\boldsymbol{R}^{2}\right\rangle}{L}=\frac{1}{D}
$$


In the limit of large $n$ or $L$, both chains are Gaussian. Therefore equality of the second moments is sufficient to obtain the identical Gaussian conformation. Thus we must have

$$
n b^{2} C_{\infty}=L / D
$$

The value of $C_{\infty}$ is known for the several chain models mentioned in the preceding section. For example we have $C_{\infty}=2$ for the freely rotating chain with tetrahedral valence angles, and $C_{\infty}=$ 6.87 for the polymethylene chain. The number of degrees of polymerization $n$ in broken chains is to be compared to the contour length $L$ of stiff chains. Since $L D$ is a dimensionless quantity, we put

$$
n=f \cdot L D
$$

and we seek an appropriate shift factor $f$ so as to give the best coincidence between the conformations of broken chains and stiff chains. From eq 7 and 8 , one obtains

$$
f=1 /(D b)^{2} C_{\infty}
$$

Thus one more relation between $D$ and $b$ is necessary in order to find the shift factor. There are several possible relations between $b$ and $D$.

\section{Case I. Persistence Length}

The persistence length of a chain is defined as the limit for large $n$ of the projection length of a chain onto the first bond, and is related to the constant $C_{\infty}$ as follows,

$$
\text { P.L. }=\frac{b}{2}\left[\lim _{n \rightarrow \infty} \frac{\left\langle\boldsymbol{R}^{2}\right\rangle}{n b^{2}}+1\right]=\frac{b}{2}\left(C_{\infty}+1\right)
$$

The persistence length for stiff chains is

$$
P . L .=1 / 2 D
$$

From eq 10 and 11 we have

and

$$
b D=1 /\left(C_{\infty}+1\right)
$$

$$
f=\left(C_{\infty}+1\right)^{2} / C_{\infty} \equiv f_{1}
$$

The value of $f$ determined by eq 13 is designated as $f_{1}$, which is equal to 4.5 for the freely rotating chain $\left(C_{\infty}=2\right)$ and to 9.02 for the polymethylene

* Jernigan and Flory have omitted the factor $1 / \sin ^{2}(\theta / 2)=1 / 6.87$ in eq 19 as in Case II and obtained $f_{3}=C_{\infty}=6.87$ instead of 10.0. This made their result of stiff chain look bad (pp 326-332 in Flory's book $^{4}$, particularly Figures 7, 8, and 9 and Figures $1,2,4$, and 5 in Jernigan and Flory's paper ${ }^{11}$ ). chain $\left(C_{\infty}=6.87\right)$.

\section{Case II. Total Chain Length}

Next we compare the length of the stiff chain and the total length of the chain molecule, and put

$$
L=n b
$$

Then we have from eq 8

$$
f=1 / D b
$$

and further from eq 9

$$
f=C_{\infty} \equiv f_{2}
$$

The value of $f$ determined by eq 15 is designated as $f_{2}$, which is equal to 2 for the freely rotating chain and to 6.87 for the polymethylene chain.

Case III. Extended Chain Length

When a chain is extended to the all-trans conformation by keeping the valence angle constant, we have the length $n b \cdot \sin (\theta / 2)$ and this is equated to $L$.

$$
L=n b \cdot \sin (\theta / 2)
$$

Then we have

$$
f=1 / D b \cdot \sin (\theta / 2)
$$

and, combined with eq 9

$$
f=C_{\infty} / \sin ^{2}(\theta / 2) \equiv f_{3}
$$

The valence angle $\theta$ is assumed either $109^{\circ}$ (tetrahedral angle) or $112^{\circ}$ which is determined from crystallographic data and is regarded as more appropriate for carbons in $-\mathrm{C}-\mathrm{C}-$ chain. The value of $f_{3}$ is equal to 3 for the freely rotating chain with the tetrahedral angle and to $10.0^{*}$ for the polymethylene chain with the valence angle $\theta=112^{\circ}$. Summarizing, we give in Table $\mathrm{I}$ the $f$-values found for Cases I, II, and III for the freely rotating chain $\left(\theta=109.5^{\circ}\right)$ and the polymethylene chains $\left(\theta=112^{\circ}\right)$. Figure 1 shows the relations $\left\langle\boldsymbol{R}^{2}\right\rangle / n b^{2}$ and $\left\langle\boldsymbol{R}^{4}\right\rangle / n^{2} b^{4} v s . n$ for the freely rotating chain with $\theta=109.5^{\circ}$,

Table I. The $f$-values for the cases I, II, and III

\begin{tabular}{lllc}
\hline & $\begin{array}{c}\text { I } \\
\text { (persistent } \\
\text { length) }\end{array}$ & $\begin{array}{c}\text { II } \\
\text { (total } \\
\text { length) }\end{array}$ & $\begin{array}{c}\text { III } \\
\text { (extended } \\
\text { length) }\end{array}$ \\
\hline $\begin{array}{l}\text { Freely rotating } \\
\text { chain }\end{array}$ & 4.5 & 2.0 & 3.0 \\
Polymethylene & 9.02 & 6.87 & 10.0 \\
\hline
\end{tabular}




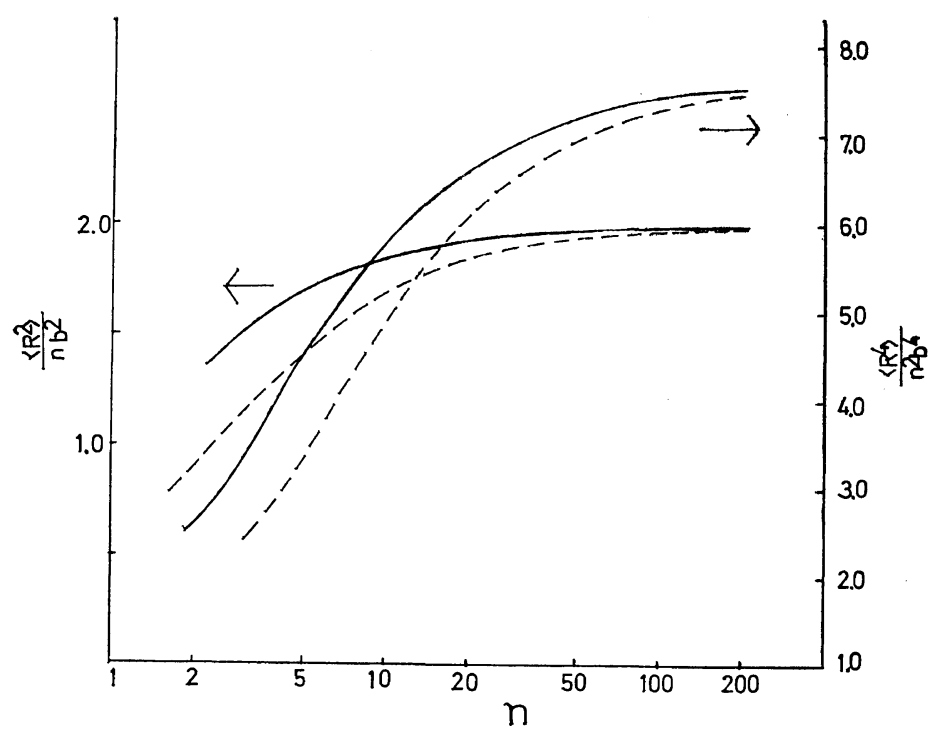

Figure 1. Comparisons of the second and the fourth moments for the freely rotationg chain and for the stiff chain with $f=3$ : —- broken chain (free rotation); ----, stiff chain.

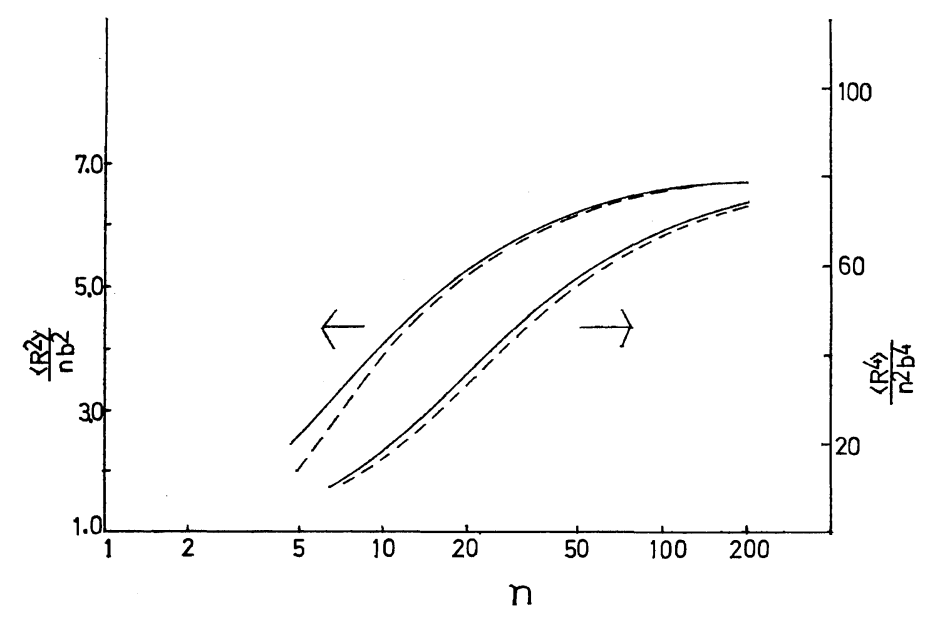

Figure 2. Comparisons of the second and the fourth moments for the chain of polymethylene type with independent rotation and for the stiff chain with $f=10.0$ : $\_$, broken chain (independent rotation); ---, stiff chain.

compared with those of the stiff chain with $f=3$ (Case III). In this figure and in what follows the full lines show the results for broken chains and the broken lines for stiff chains. Figures 2 and 3 show similar plots for the polymethylene chain of model (c) (Figure 2) and of model (d) (Figure 3) with $f=10.0$ (Case III).

The agreement of the two conformations is
Table II. The best-fitted $f$-values

\begin{tabular}{lccc}
\hline & $\left\langle\boldsymbol{R}^{2}\right\rangle / n b^{2}$ & $\left\langle\boldsymbol{R}^{4}\right\rangle / n^{2} b^{4}$ & Average \\
\hline $\begin{array}{c}\text { Freely-rotating chain } \\
\text { Chain with interde- } \\
\text { pendent rotation of }\end{array}$ & 1.50 & 1.83 & 1.66 \\
$\begin{array}{c}\text { polymethylene type } \\
\text { Chain with independent } \\
\text { rotation of poly- } \\
\text { methylene type }\end{array}$ & 10.10 & 10.87 & 10.49 \\
\hline
\end{tabular}




\section{H. Maeda, N. Saito, and H. Stockmayer}

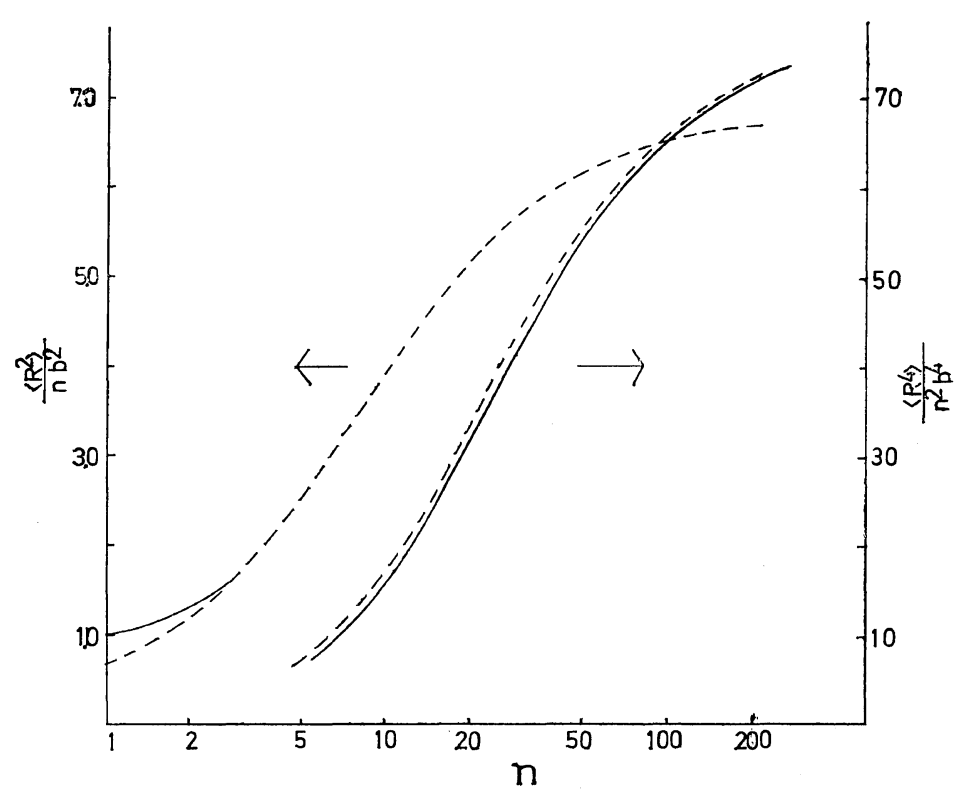

Figure 3. Comparisons of the second and the fourth moments for the chain of polymethylene type with interdependent rotation and for the stiff chain with $f=10.0$ : $\quad$, broken chain (interdependent rotation); ----, stiff chain.

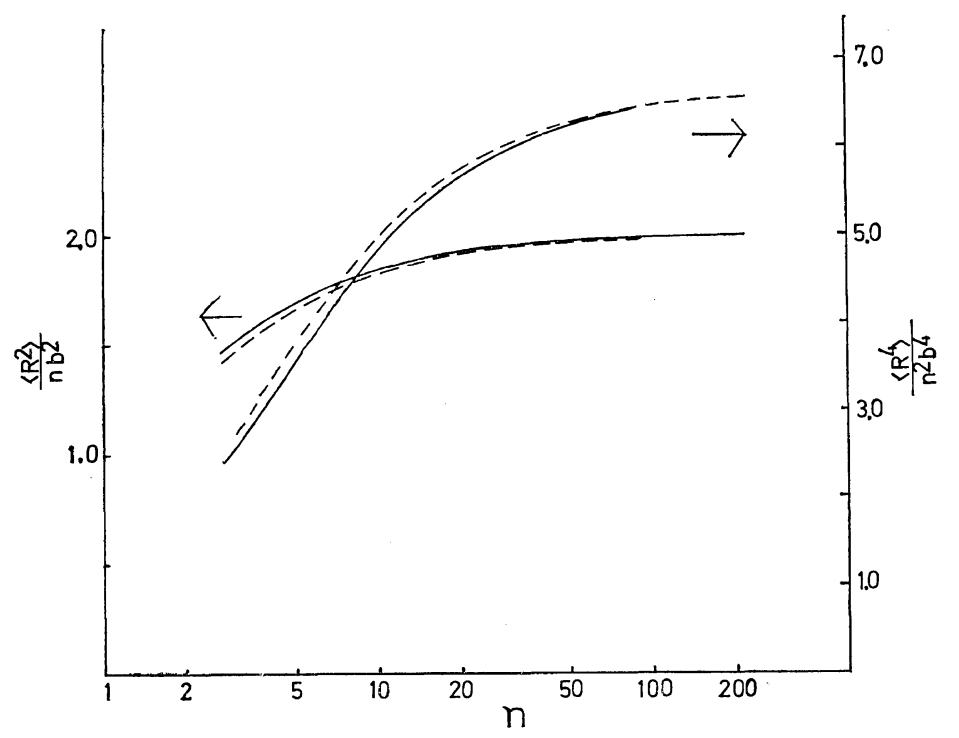

Figure 4. Comparisons of the second and the fourth moments for the freely rotating chain and for stiff chain with $f=1.66$ : — broken chain (freely rotating); ----, stiff chain.

not particularly good. One can, however, determine $f$-values which make the best coincidence for each figure. These $f$-values are listed in Table II. On comparing Table II with Table I, we see that we cannot choose one of the cases I, II, and III as always the best for the determination of $f$ values, although Case III is clearly best for polymethylene. One also notices that 


\section{Conformations of Short Broken Chains and Stiff Chains}

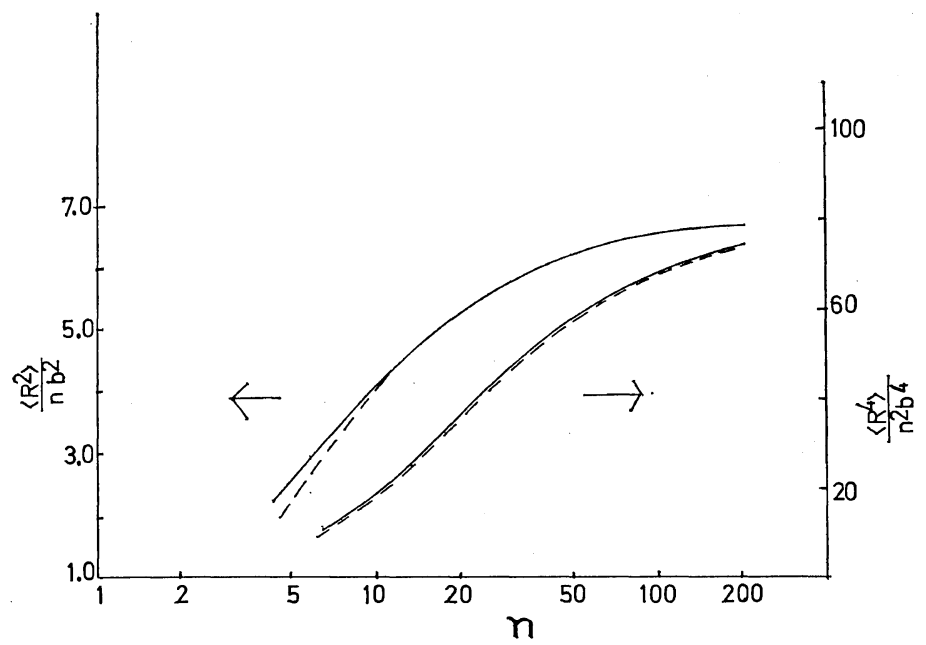

Figure 5. Comparisons of the second and the fourth moments for the chain of polymethylene type with independent rotation and for the stiff chain with $f=9.18$ : - , broken chain (independent rotation); ----, stiff chain.

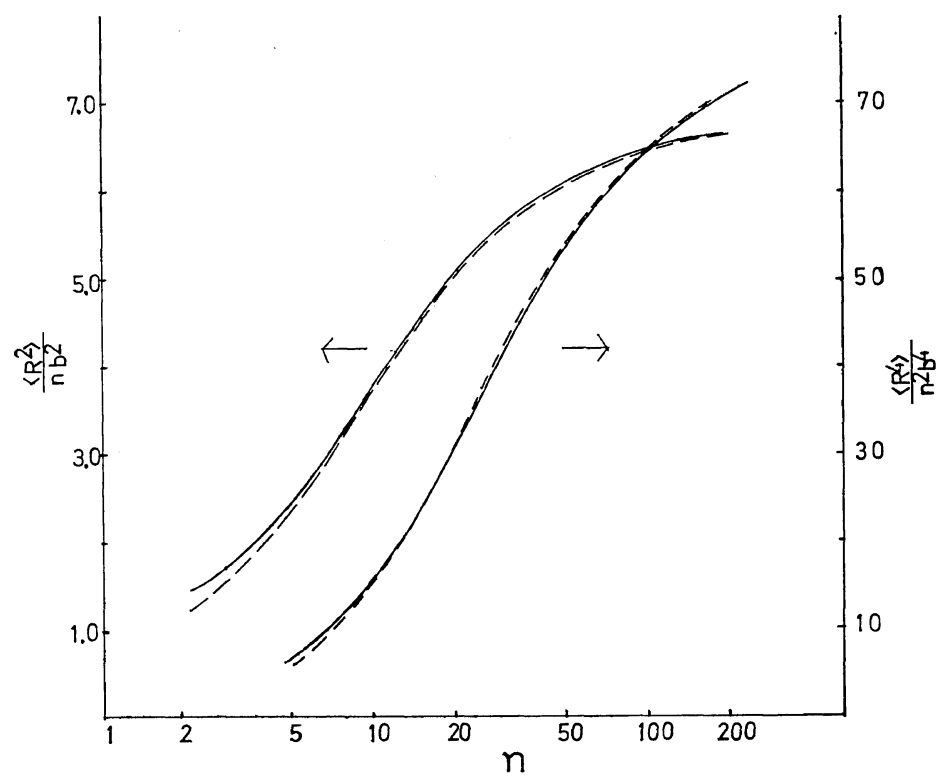

Figure 6. Comparisons of the second and the fourth moments for the chain of polymethylene type with interdependent rotation and for the stiff chain with $f=10.49$ : —

the $f$-values listed in Table II are almost equal for the second and the fourth moments both in the freely rotating chain and in the polymethylene chain, so we may try the average $f$ value. Figure 4 show the coincidence of the two conformations for the freely rotating chain with $f=1.66$. Figures 5 and 6 show a similar result for polymethylene chains. The former (Figure 5) is for the polymethylene chain with independent rotations and $f=9.18$ and the latter (Figure 6) is for the polymethylene chain with interdependent rotations and $f=10.49$. The agreement is quite good. 


\section{DISCUSSION}

We have shown in the preceding sections that the conformation of a short broken chain can be quite well represented by that of a stiff chain, provided that an adequate shift factor $f$ is employed. The agreement shown in Figures 4, 5 , and 6 is, of course, only over limited range of $n$, as large as $n>15$. We may conclude that the stiff-chain model for short broken chains for $n>15$ is useful for discussing the behavior of oligomers in solution, especially their hydrodynamical properties.

One may question whether the agreement of the second and the fourth moments is sufficient to establish complete agreement of the two conformations; and further one may imagine that the cases of the freely rotating chain and the polymethylene chain may be fortunate ones. However, shift factors found ${ }^{16}$ according to Case III also give rather good results for second moments in the comparison of stiff chains with realistic interdependent-rotation models for polyoxyethylene and enen for polyoxymethylene, despite the predominance of gauche rotational states in the latter chain. Yet, in the case of poly(dimethylsiloxane), the best shift factor is considerably smaller than those based on either Case II or Case III. Moreover, Flory ${ }^{4}$ gives an example for which no shift factor is appropriate; the characteristic ratio of the alternating racemic polymer of DL-alanine actually decreases with increasing chain length.

We may conclude that the stiff or wormlike chain will very often serve as a useful and valid approximation to the conformational behavior of real chains, unless these are extremely short; and that in many instance the best shift actor will be not very different from that found iccording to the method of Case III.

Acknowledgment. The authors would like to thank Dr. Jernigan and Prof. P. J. Flory of Stanford University for providing us with the numerical data on the polymethylene molecule.

\section{REFERENCES}

1. N. Saitô, "Introduction to Polymer Physics", Revised ed, Syokabo, Tokyo, 1966, p 432.

2. C. M. Tchen, J. Chem. Phys., 20, 214 (1952).

3. M. V. Volkenstein, "Configurational Statistics of Polymeric Chains", S. N. Timasheff and M. J. Timasheff, Translators, Interscience Publishers, Inc., New York, N.Y., 1963.

4. P. J. Flory, "Statistical Mechanics of Chain Molecules", Interscience Publishers, Inc., New York, N.Y., 1969.

5. N. Saitô, K. Okano, S. Iwayanagi, and T. Hideshima, "Solid State Physics", Vol. 14, F. Seitz and D. Turnbull, Ed., Acad. Press, New York, N.Y., 1963, p 343.

6. O. Kratky and G. Porod, Rec. Trav. Chim., 68, 1106 (1949).

7. H. E. Daniels, Proc. Roy. Soc. Edinburgh, 63A, 290 (1952).

8. J. J. Hermans and R. Ullmans, Physica., 18, 951 (1952).

9. S. Heine, O. Kratky, G. Porod, and P. J. Schmitz, Makromol. Chem., 46, 682 (1961).

10. N. Saitô, Y. Yunoki, and K. Takahashi, J. Phys. Soc. Japan, 22, 219 (1967).

11. R. L. Jernigan and P. J. Flory, J. Chem. Phys., 50, 4165 and 4178 (1969).

12. S. Oka, Proc. Phys.-Math. Soc. Japan, 24, 657 (1942).

13. K. Nagai, J. Chem. Phys., 45, 838 (1966).

14. K. Nagai, J. Chem. Phys., 48, 5646 (1968).

15. S. Lifson, J. Chem. Phys., 30, 964 (1959).

16. W. H. Stockmayer, unpublished calculations. 